\title{
The spectral mean value for linear forms in twisted coefficients of cusp forms
}

\author{
by \\ Wenzhi Luo (Berkeley, Calif.)
}

1. Introduction and statement of results. Let $\left\{u_{j}(z)\right\}_{1}^{\infty}$ be an orthonormal basis of Maass cusp forms for the modular group $\Gamma=\mathrm{SL}_{2}(\mathbb{Z})$. Suppose that $u_{j}(x+i y)$ is either even or odd in $x$. Thus $u_{j}(z)$ is an eigenfunction of the Laplace operator with eigenvalue $\lambda_{j}=s_{j}\left(1-s_{j}\right)$, where $s_{j}=1 / 2+i t_{j}$ with $t_{j}>0$, and it has the Fourier expansion

$$
u_{j}(z)=2 y^{1 / 2} \sum_{n=1}^{\infty} \varrho_{j}(n) K_{i t_{j}}(2 \pi n y) \cos (2 \pi n x)
$$

or

$$
u_{j}(z)=2 y^{1 / 2} \sum_{n=1}^{\infty} \varrho_{j}(n) K_{i t_{j}}(2 \pi n y) \sin (2 \pi n x)
$$

according to whether $u_{j}(z)$ is even or odd, where $K_{\nu}$ is the $K$-Bessel function. The Weyl law (proved by A. Selberg [14], see also [4])

$$
\sharp\left\{j: t_{j} \leq T\right\} \sim T^{2} / 12
$$

shows that there are infinitely many linearly independent cusp forms but none of them have ever been constructed. The Fourier coefficients $\varrho_{j}(n)$ are the subject of various studies. There are still basic questions to be answered, such as what is the order of magnitude of $\varrho_{j}(n)$ ? From the asymptotic formula (due to Rankin [12] and Selberg [13])

$$
\sum_{n \leq N}\left(\cosh \pi t_{j}\right)^{-1}\left|\varrho_{j}(n)\right|^{2} \sim 12 \pi^{-2} N
$$

and the formula (due to Kuznetsov [8])

$$
\sum_{t_{j} \leq T}\left(\cosh \pi t_{j}\right)^{-1}\left|\varrho_{j}(n)\right|^{2} \sim \pi^{-2} T^{2}
$$

it follows that $\left(\cosh \pi t_{j}\right)^{-1 / 2} \varrho_{j}(n)$ is bounded on average in $n$ and $t_{j}$. The oscillatory behavior of $\varrho_{j}(n)$ is revealed in the large sieve type inequality of 
H. Iwaniec [6]

$$
\sum_{t_{j} \leq T}\left(\cosh \pi t_{j}\right)^{-1}\left|\sum_{n \leq N} a_{n} \varrho_{j}(n)\right|^{2} \ll\left(T^{2}+N^{1+\varepsilon}\right) \sum_{n \leq N}\left|a_{n}\right|^{2} .
$$

Another large sieve type inequality for the twisted coefficients $\varrho_{j}(n) n^{i t_{j}}$ was established by J.-M. Deshouillers and H. Iwaniec. They proved, among other things, that

$$
\sum_{t_{j} \leq T}\left(\cosh \pi t_{j}\right)^{-1}\left|\sum_{n \leq N} a_{n} \varrho_{j}(n) n^{i t_{j}}\right|^{2} \ll\left(T^{2}+N^{2}\right)(N T)^{\varepsilon} \sum_{n \leq N}\left|a_{n}\right|^{2}
$$

for arbitrary complex numbers $a_{n}$ (see Theorem 6 of [2]). Estimates for the linear forms of type (7) are used to prove the non-vanishing of certain automorphic $L$-functions at the special points $s=s_{j}$ which occur in the Phillips-Sarnak theory of deformation of groups [11]. The strongest results in this connection are established in [9].

In this paper we shall improve upon (7) substantially.

THEOREM 1. For any complex numbers $a_{n}$ we have

$$
\begin{aligned}
\sum_{t_{j} \leq T}\left(\cosh \pi t_{j}\right)^{-1} \mid \sum_{n \leq N} & \left.a_{n} \varrho_{j}(n) n^{i t_{j}}\right|^{2} \\
& \ll\left(T^{2}+T^{3 / 2} N^{1 / 2}+N^{5 / 4}\right)(N T)^{\varepsilon} \sum_{n \leq N}\left|a_{n}\right|^{2} .
\end{aligned}
$$

The implied constant depends on $\varepsilon$ only.

This result is stronger than (7) if $N \gg T$; however, it is not the best possible. In view of (6) one might expect the same bound to hold true for (8), but we cannot prove it along the lines of this paper. In order to understand the difference between (6) and (8) and the depth of (8) let us note that (6) extends to the corresponding contribution from the continuous spectrum while (8) would be false if such contribution was included. Since our approach to (8) appeals to the complete spectral resolution of the Laplace operator via Kuznetsov's formula we have to treat the dominating terms from the continuous spectrum with great care. The arguments are subtle. We shall identify the terms from Eisenstein series with a part of sums of Kloosterman sums in the Kuznetsov formula by delicate analysis and then cancel them out. This correspondence is not of an algebraic or combinatorial type, and it seems to be a novelty in the spectral topics of automorphic forms.

Recently M. Jutila [7] has generalized (6) by allowing perturbations of type $e\left(f\left(n, t_{j}\right)\right)$, where $f$ is a smooth function which has rather small derivatives. However, our result cannot be derived by his method. 
The special feature of the twisting factor $n^{i t_{j}}$ is better appreciated in the context of the Hecke $L$-functions

$$
L_{j}(s)=\sum_{n=1}^{\infty} \lambda_{j}(n) n^{-s},
$$

where $\lambda_{j}(n)=\varrho_{j}(n) / \varrho_{j}(1)$ are the eigenvalues of the Hecke operator (see [15]). The series converges absolutely in $\Re s>1$, it has analytic continuation to an entire function and it satisfies one of the functional equations

$$
\begin{gathered}
\theta_{j}(s) L_{j}(s)=\theta_{j}(1-s) L_{j}(1-s), \\
\theta_{j}(1+s) L_{j}(s)=-\theta_{j}(2-s) L_{j}(1-s),
\end{gathered}
$$

according to the parity of $u_{j}(z)$, where

$$
\theta_{j}(s)=\pi^{-s} \Gamma\left(\frac{s+i t_{j}}{2}\right) \Gamma\left(\frac{s-i t_{j}}{2}\right) .
$$

Here the presence of two gamma factors is intrinsic for $L$-functions attached to $\mathrm{GL}_{2}$ automorphic forms. However, at the special point $s=s_{j}=1 / 2+i t_{j}$ the second factor is constant in $t_{j}$, so that $L_{j}\left(s_{j}\right)$ behaves analytically like an $L$-function for a character. The key point is that $L_{j}\left(s_{j}\right)$ can be well approximated by partial sums of length $N \sim t_{j}^{1 / 2}$, which is considerably shorter than $N \sim t_{j}$, required for general fixed $s$. Using this approximation one can infer by Theorem 1 the following power moment estimates:

$$
\sum_{t_{j} \leq T}\left|L_{j}\left(s_{j}\right)\right|^{4} \ll T^{2+\varepsilon}
$$

and

$$
\sum_{t_{j} \leq T}\left|L_{j}\left(s_{j}\right)\right|^{8} \ll T^{5 / 2+\varepsilon} .
$$

The last bound should be $T^{2+\varepsilon}$ but we cannot prove it at present. This would be a close analogy to a result of M. N. Huxley [5] for Dirichlet $L$-functions.

For the proof of (8) we shall deal with a smoothed sum

$$
S(\mathcal{A})=\sum_{j} \omega\left(t_{j}\right)\left|\sum_{n} a_{n} \varrho_{j}(n) n^{i t_{j}}\right|^{2},
$$

where

$$
\omega(t)=2 \frac{\sinh (\pi-2 \delta) t}{\sinh 2 \pi t}, \quad 2 \delta=T^{-1}
$$

and $\mathcal{A}=\left(a_{n}\right)$ is a finite sequence of real numbers for $N<n \leq 2 N$. We denote the $l_{2}$-norm by

$$
\|\mathcal{A}\|^{2}=\sum a_{n}^{2}
$$


We shall simultaneously consider the contribution from the Eisenstein series

$$
E(z, s)=y^{s}+\varphi(s) y^{1-s}+2 y^{1 / 2} \sum_{n=1}^{\infty} \eta_{t}(n) K_{i t}(2 \pi n y) \cos (2 \pi n x)
$$

on the line $s=1 / 2+i t$. Here the Fourier coefficients are given explicitly by $\eta_{t}(n)=\eta_{t}(1) \tau_{t}(n)$, where $\eta_{t}(1)=2 \pi^{s} \zeta(2 s)^{-1} \Gamma(s)^{-1}$ and

$$
\tau_{t}(n)=\sum_{d_{1} d_{2}=n}\left(d_{1} / d_{2}\right)^{i t} .
$$

The corresponding contribution from the continuous spectrum is

$$
T(\mathcal{A})=\frac{1}{4 \pi} \int_{-\infty}^{\infty} \omega(t)\left|\sum_{n} a_{n} \eta_{t}(n) n^{i t}\right|^{2} d t,
$$

where $(4 \pi)^{-1} d t$ is the spectral measure. For this we shall prove directly the following

Proposition 1. If $N \gg T$ we have

$$
T(\mathcal{A})=(\pi \tan \delta)^{-1} \sum_{m} \sum_{n} a_{m} a_{n} \sigma(m, n)+O\left(\left(N+T^{2}\right) N^{\varepsilon}\|\mathcal{A}\|^{2}\right),
$$

where

$$
\sigma(m, n)=\sum_{r=1}^{\infty} r^{-2} S(0, m ; r) S(0, n ; r)
$$

and $S(0, m ; r)$ is the Ramanujan sum.

Using the expression

$$
S(0, m ; r)=\sum_{d \mid(m, r)} d \mu(r / d)
$$

one can easily execute the summation over $r$ in $\sigma(m, n)$ getting a finite expression

$$
\sigma(m, n)=\frac{\zeta(2)}{\zeta(4)} \sum_{\alpha \gamma \mid m} \sum_{\beta \gamma \mid n} \mu(\alpha \beta) \prod_{p \mid \alpha \beta}\left(p+p^{-1}\right)^{-1} .
$$

Proposition 2. If $N \gg T$ we have

$$
\begin{aligned}
S(\mathcal{A})+T(\mathcal{A})= & (\pi \tan \delta)^{-1} \sum_{m} \sum_{n} a_{m} a_{n} \sigma(m, n) \\
& +O\left(\left(T^{2}+T^{3 / 2} N^{1 / 2}+N^{3 / 2} T^{-1}\right) N^{\varepsilon}\|\mathcal{A}\|^{2}\right) .
\end{aligned}
$$

Combining (20) with (21) we obtain

$$
S(\mathcal{A}) \ll\left(T^{2}+T^{3 / 2} N^{1 / 2}+N^{3 / 2} T^{-1}\right) N^{\varepsilon}\|\mathcal{A}\|^{2} .
$$


Since $S(\mathcal{A})$ is increasing in $T$ one may replace $T$ above by $T+N^{1 / 2}$ getting

$$
S(\mathcal{A}) \ll\left(T^{2}+T^{3 / 2} N^{1 / 2}+N^{5 / 4}\right) N^{\varepsilon}\|\mathcal{A}\|^{2} .
$$

This, of course, implies (8) if $N>T$. For $N \leq T$ we infer (8) from (7).

This work is part of my Ph.D. dissertation under the guidance of Professor H. Iwaniec. I wish to express my gratitude to him for introducing me to this fascinating area of research and providing valuable advice and encouragement throughout this work.

2. An application of Kuznetsov's formula. For $m, n \in \mathbb{Z}$, the Kloosterman sum is defined as

$$
S(m, n ; c)=\sum_{a d \equiv 1 \bmod c} e\left(\frac{m a+n d}{c}\right) .
$$

We shall transform $S(\mathcal{A})+T(\mathcal{A})$ into a sum of Kloosterman sums $S(m, n ; c)$ by an appeal to the Kuznetsov formula [8]

$$
\begin{aligned}
\sum_{j} \widehat{f}\left(t_{j}\right) \varrho_{j}(m) \bar{\varrho}_{j}(n) & +\frac{1}{4 \pi} \int_{-\infty}^{\infty} \widehat{f}(t) \eta_{t}(m) \bar{\eta}_{t}(n) d t \\
& =\delta_{m n} f_{0}+\sum_{c} c^{-1} S(m, n ; c) f_{A}\left(\frac{4 \pi \sqrt{m n}}{c}\right) .
\end{aligned}
$$

Here $f(y)$ is a smooth function for $y \geq 0$ satisfying the growth conditions $f(y) \ll y$ as $y \rightarrow 0$ and $f^{(\mu)}(y) \ll y^{-3}$ as $y \rightarrow \infty$, for $\mu=0,1,2,3$, and

$$
\begin{aligned}
f_{0} & =\frac{1}{2 \pi} \int_{0}^{\infty} J_{0}(y) f(y) d y \\
\widehat{f}(t) & =\frac{\pi i}{\sinh 2 \pi t} \int_{0}^{\infty}\left(J_{2 i t}(y)-J_{-2 i t}(y)\right) f(y) y^{-1} d y \\
f_{A}(x) & =\int_{1}^{\infty} u x J_{0}(u x) \int_{0}^{\infty} J_{0}(u y) f(y) d y d u
\end{aligned}
$$

where $J_{\nu}(x)$ is the $J$-Bessel function.

We shall also use an analogous formula for the Fourier coefficients of holomorphic cusp forms

$$
f_{j k}(z)=\sum_{n=1}^{\infty} a_{j k}(n) e(n z)
$$

where $\left\{f_{j k}(z)\right\}_{j=1}^{\theta_{k}}$ is an orthonormal basis for the space of cusp forms of 
weight $k$. We then have

$$
\begin{aligned}
& \sum_{c} c^{-1} S(m, n ; c) f_{B}\left(\frac{4 \pi \sqrt{m n}}{c}\right) \\
&=\delta_{m n} f_{0}+\sum_{k>0,2 \mid k} \frac{i^{k}(k-1) ! \tilde{f}(k-1)}{\pi(4 \pi \sqrt{m n})^{k-1}} \sum_{j} \bar{a}_{j k}(m) a_{j k}(n),
\end{aligned}
$$

where

$$
\begin{gathered}
\widetilde{f}(k-1)=\int_{0}^{\infty} f(x) J_{k-1}(x) \frac{d x}{x}, \\
f_{B}(x)=\int_{0}^{1} u x J_{0}(u x) \int_{0}^{\infty} J_{0}(u y) f(y) d y d u .
\end{gathered}
$$

Notice that by Hankel's inversion formula $f$ splits into $f=f_{A}+f_{B}$.

As in [2] we shall apply (23) for the test function

$$
f(y)=\pi^{-1} y(\sinh \beta) e^{i y \cosh \beta},
$$

with

$$
\beta=\frac{1}{2}\left|\log \frac{m}{n}\right|+i \delta
$$

Therefore

$$
\begin{aligned}
f\left(\frac{4 \pi \sqrt{m n}}{c}\right)= & \frac{2}{c}(|m-n| \cos \delta+i(m+n) \sin \delta) \\
& \times e\left(\frac{m+n}{c} \cos \delta\right) \exp \left(-2 \pi \frac{|m-n|}{c} \sin \delta\right) .
\end{aligned}
$$

It has been shown in [2] that

$$
f_{0}=\frac{-1}{2 \pi^{2}} \cdot \frac{\cosh \beta}{(\sinh \beta)^{2}} \quad \text { and } \quad \widehat{f}(t)=2 \frac{\sinh (\pi+2 i \beta) t}{\sinh 2 \pi t} .
$$

Therefore

$$
\Re \widehat{f}(t)=\omega(t) \cos \left(t \log \frac{m}{n}\right) .
$$

From these evaluations we infer by (23) the identity

$$
S(\mathcal{A})+T(\mathcal{A})=\frac{\cos \delta}{2 \pi^{2} \sin ^{2} \delta}\|\mathcal{A}\|^{2}+\Re P(\mathcal{A}),
$$

where

$$
P(\mathcal{A})=\sum_{m, n} a_{m} a_{n} \sum_{c} c^{-1} S(m, n ; c) f_{A}\left(\frac{4 \pi \sqrt{m n}}{c}\right) .
$$


It turns out to be more convenient to work with $f_{B}$ than with $f_{A}$. Therefore we write $f_{A}=f-f_{B}$ and put

$$
\begin{aligned}
Q(\mathcal{A}) & =\sum_{m, n} a_{m} a_{n} \sum_{c} c^{-1} S(m, n ; c) f\left(\frac{4 \pi \sqrt{m n}}{c}\right), \\
R(\mathcal{A}) & =\sum_{m, n} a_{m} a_{n} \sum_{c} c^{-1} S(m, n ; c) f_{B}\left(\frac{4 \pi \sqrt{m n}}{c}\right) .
\end{aligned}
$$

Accordingly $P(\mathcal{A})$ splits into

$$
P(\mathcal{A})=Q(\mathcal{A})-R(\mathcal{A})
$$

The next sections will be devoted separately to the treatment of the above two terms.

3. Evaluation of $Q(\mathcal{A})$. We split

$$
Q(\mathcal{A})=2 Q_{1}(\mathcal{A}) \cos \delta+2 i Q_{2}(\mathcal{A}) \sin \delta
$$

where

$$
\begin{aligned}
Q_{1}(\mathcal{A})= & \sum_{m, n} a_{m} a_{n}|m-n| \sum_{c} c^{-2} S(m, n ; c) \\
& \times e\left(\frac{m+n}{c} \cos \delta\right) \exp \left(\frac{-2 \pi|m-n|}{c} \sin \delta\right),
\end{aligned}
$$

and

$$
\begin{aligned}
Q_{2}(\mathcal{A})= & \sum_{m, n} a_{m} a_{n}(m+n) \sum_{c} c^{-2} S(m, n ; c) \\
& \times e\left(\frac{m+n}{c} \cos \delta\right) \exp \left(\frac{-2 \pi|m-n|}{c} \sin \delta\right) .
\end{aligned}
$$

First we shall show that

$$
Q_{2}(\mathcal{A}) \ll \delta^{-1} N^{1+\varepsilon}\|\mathcal{A}\|^{2},
$$

which is absorbed in (21). Since large $c$ 's contribute little (use Weil's bound for Kloosterman sums) we can restrict the summation to $c \leq N^{8}$. We split the remaining range into dyadic intervals $C<c \leq 2 C \leq N^{8}$ and denote the corresponding contribution by $Q_{2 C}(\mathcal{A})$. In $Q_{2 C}(\mathcal{A})$ we split the summation over $m, n$ into boxes $B=I \times J$ where $I, J$ are subintervals of $[N, 2 N]$ of equal length $\Delta=N K^{-1}$ with $K=\max \left(1,\left[\delta C^{-1} N^{1-\varepsilon}\right]\right)$. Let $Q_{2 I J}(\mathcal{A})$ denote the corresponding partial sum of $Q_{2 C}(\mathcal{A})$. If $I, J$ are neither equal nor adjacent then for $(m, n) \in B$ we have $|m-n|>\Delta \geq \delta^{-1} C N^{\varepsilon}$, whence trivially

$$
Q_{2 I J}(\mathcal{A}) \ll N^{-10}\|\mathcal{A}\|^{2} .
$$

All these boxes contribute $O\left(K^{2} N^{-10}\|\mathcal{A}\|^{2}\right)$. The number of remaining boxes to be considered is at most $3 K$. For these we will apply the mean 
value theorem (see [10])

$$
\sum_{d \bmod c}\left|\sum_{n=M+1}^{M+N} a_{n} e\left(\frac{d n}{c}\right)\right|^{2} \ll(c+N) \sum_{n=M+1}^{M+N}\left|a_{n}\right|^{2} .
$$

To this end, we separate the variables $m$ and $n$ by using the Fourier integral

$$
\exp (-2 \pi|t|)=\frac{1}{\pi} \int_{-\infty}^{\infty} e(\xi t)\left(1+\xi^{2}\right)^{-1} d \xi .
$$

We infer, by opening the Kloosterman sum, that

$$
\begin{aligned}
& Q_{2 I J}(\mathcal{A})=\sum_{n, m \in B} a_{n} a_{m}(m+n) \\
& \quad \times \sum_{C<c \leq 2 C} c^{-2} S(m, n ; c) e\left(\frac{m+n}{c} \cos \delta\right) \exp \left(\frac{-2 \pi|m-n|}{c} \sin \delta\right) \\
& =\frac{1}{\pi} \sum_{C<c \leq 2 C} c^{-2} \sum_{a d \equiv 1 \bmod c} \int_{-\infty}^{\infty}\left(1+\xi^{2}\right)^{-1} \\
& \quad \times \sum_{n, m \in B} a_{n} a_{m}(m+n) e\left(\frac{m a+n d}{c}\right) e\left(\frac{m+n}{c} \cos \delta\right) e\left(\xi \frac{m-n}{c} \sin \delta\right) d \xi \\
& \ll N C^{-1}(C+\Delta)\left(\sum_{m \in I} a_{m}^{2}\right)^{1 / 2}\left(\sum_{n \in J} a_{n}^{2}\right)^{1 / 2} .
\end{aligned}
$$

Summing over the boxes yields

$$
Q_{2 C}(\mathcal{A}) \ll N C^{-1}(C+\Delta)\|\mathcal{A}\|^{2} \ll \delta^{-1} N^{1+\varepsilon}\|\mathcal{A}\|^{2},
$$

whence (32) follows.

Next we modify $Q_{1}(\mathcal{A})$ by applying the approximation

$$
e\left(\frac{m+n}{c} \cos \delta\right)=e\left(\frac{m+n}{c}\right)(1+E),
$$

where

$$
E=e\left(-2 \frac{m+n}{c} \sin ^{2} \frac{\delta}{2}\right)-1 \ll \delta^{2} c^{-1} N .
$$

One can show that the error term resulting from $E$ is admissible. Indeed, the same argument which was applied above for $Q_{2}(\mathcal{A})$ works here except that for separating the variables we use the Fourier transform

$$
h(t)=|t| e^{-2 \pi|t|}=\int_{-\infty}^{\infty} \widehat{h}(s) e(-s t) d s,
$$


with $\widehat{h}(s)=\left(1-s^{2}\right) /\left(2 \pi^{2}\left(1+s^{2}\right)^{2}\right)$ in place of (34) and we expand $E$ into power series

$$
E=\sum_{k=1}^{\infty} \frac{1}{k !}\left(\frac{-2(m+n)}{c} \sin ^{2} \frac{\delta}{2}\right)^{k}
$$

if $C>\delta^{2} N^{1+\varepsilon}$ in which case the series converges rapidly. We obtain

$$
Q_{1}(\mathcal{A})=Q_{0}(\mathcal{A})+O\left(N^{1+\varepsilon}\|\mathcal{A}\|^{2}\right)
$$

where

$$
\begin{aligned}
Q_{0}(\mathcal{A})= & \sum_{m \neq n} a_{m} a_{n}|m-n| \\
& \times \sum_{c} c^{-2} S(m, n ; c) e\left(\frac{m+n}{c}\right) \exp \left(\frac{-2 \pi|m-n|}{c} \sin \delta\right) .
\end{aligned}
$$

Define

$$
E(m, n)=\sum_{c=1}^{\infty} c^{-2} S(m, n ; c) e\left(\frac{m+n}{c}\right) \exp \left(\frac{-y}{c}\right),
$$

with $y=2 \pi|m-n| \sin \delta$ in mind. We write

$$
S(m, n ; c) e\left(\frac{m+n}{c}\right)=\sum_{d \bmod c} e_{c}((1-\bar{d}) m+(1-d) n),
$$

and split the summation by fixing the values $(d-1, c)=q$, say. Thus $c=q r$ and $d=1-q \bar{s}$, where $s$ ranges over classes $\bmod r$ such that $(s(q-s), r)=1$. We obtain

$$
S(m, n ; c) e\left(\frac{m+n}{c}\right)=\sum_{s \bmod r,(s(q-s), r)=1} e_{r}(\bar{s} n+\overline{q-s} m) .
$$

Hence

$$
\begin{aligned}
& E(m, n) \\
& =\sum_{q r \leq N^{8}}(q r)^{-2} \sum_{s \bmod r,(s(q-s), r)=1} e_{r}(\bar{s} n+\overline{q-s} m) \exp \left(\frac{-y}{q r}\right)+O\left(N^{\varepsilon-4}\right) \\
& =E_{0}(m, n)+E_{1}(m, n)+O\left(N^{\varepsilon-4}\right),
\end{aligned}
$$

say, where $E_{0}(m, n)$ denotes the partial sum restricted by $r<X$ and $E_{1}(m, n)$ is the remaining sum over $r \geq X$. Here $X(\leq N)$ is a positive parameter which will be chosen optimally later. Accordingly we have

$$
Q_{0}(\mathcal{A})=Q_{00}(\mathcal{A})+Q_{01}(\mathcal{A})+O\left(\|\mathcal{A}\|^{2}\right)
$$

where

$$
Q_{00}(\mathcal{A})=\sum_{m \neq n} a_{m} a_{n}|m-n| \sum_{r<X} r^{-2} \sum_{q r \leq N^{8}} q^{-2}
$$




$$
\times \sum_{s \bmod r,(s(q-s), r)=1} e_{r}(\bar{s} n+\overline{q-s} m) \exp \left(\frac{-y}{q r}\right)
$$

with $y=2 \pi|m-n| \sin \delta$, and $Q_{01}(\mathcal{A})$ is given by the same expression as above except that the condition $r<X$ is replaced by $r \geq X$. We will extract the main term from $Q_{00}(\mathcal{A})$ and show that $Q_{01}(\mathcal{A})$ is small. Indeed, by the large sieve inequality (see [10]):

$$
\sum_{r \leq R} \sum_{s \bmod r}^{*}\left|\sum_{M+1 \leq n \leq M+N} a_{n} e\left(\frac{s n}{r}\right)\right|^{2} \ll\left(R^{2}+N\right) \sum_{M+1 \leq n \leq M+N}\left|a_{n}\right|^{2},
$$

using an argument similiar to that applied for $Q_{2}(\mathcal{A})$ to separate variables, we deduce that

$$
Q_{01}(\mathcal{A}) \ll N^{1+\varepsilon}\left(1+\delta^{-1} X^{-1}\right)\|\mathcal{A}\|^{2} .
$$

Now we evaluate $Q_{00}(\mathcal{A})$. We execute summation over $q$ by splitting into progressions:

$\sum_{(q-s, r)=1} q^{-2} \exp \left(\frac{-y}{q r}\right) e_{r}(\overline{q-s} m)=\sum_{v \bmod r} e_{r}(\bar{v} m) \sum_{q \equiv s+v \bmod r} q^{-2} \exp \left(\frac{-y}{q r}\right)$ and then apply the Euler-Maclaurin formula for the innermost sum getting

$$
\begin{aligned}
r^{-1} \int_{0}^{\infty} t^{-2} \exp \left(\frac{-y}{t r}\right) d t+\int_{0}^{\infty} \psi\left(\frac{t-s-v}{r}\right) d t^{-2} \exp \left(\frac{-y}{t r}\right) \\
=y^{-1}+\int_{0}^{\infty}\left[\psi\left(\frac{t-s-v}{r}\right)-\psi\left(\frac{-s-v}{r}\right)\right] d t^{-2} \exp \left(\frac{-y}{t r}\right) .
\end{aligned}
$$

We obtain

$$
\begin{aligned}
& Q_{00}(\mathcal{A}) \\
& =(2 \pi \sin \delta)^{-1} \sum_{m \neq n} a_{m} a_{n} \sum_{r<X} r^{-2} S(0, m ; r) S(0, n ; r) \\
& \quad+\sum_{m \neq n} a_{m} a_{n}|m-n| \sum_{r<X} r^{-2} \sum_{s, v \bmod r} e\left(\frac{\bar{s} n+\bar{v} m}{r}\right) \\
& \quad \times \int_{0}^{\infty}\left[\psi\left(\frac{t-s-v}{r}\right)-\psi\left(\frac{-s-v}{r}\right)\right] d t^{-2} \exp \left(\frac{-2 \pi|m-n| \sin \delta}{t r}\right) \\
& =Q_{000}(\mathcal{A})+Q_{001}(\mathcal{A}),
\end{aligned}
$$

say. We drop the restriction $r<X$ in $Q_{000}(\mathcal{A})$ and estimate the tail using 
the trivial bound $|S(0, m ; r)| \leq(m, r) d(m)$ to get

(40) $Q_{000}(\mathcal{A})=(2 \pi \sin \delta)^{-1} \sum_{m \neq n} a_{m} a_{n} \sigma(m, n)+O\left(\delta^{-1} X^{-1} N^{1+\varepsilon}\|\mathcal{A}\|^{2}\right)$.

Using the large sieve inequality (38), the estimate

$$
\sum_{s \bmod r}\left|\psi\left(\frac{t+s}{r}\right)-\psi\left(\frac{s}{r}\right)\right| \ll \min (t, r) r^{\varepsilon},
$$

and the argument similar to that applied for $Q_{2}(\mathcal{A})$ to separate variables we infer that

$$
Q_{001}(\mathcal{A}) \ll\left(X T+T^{3 / 2} N^{1 / 2}\right) N^{\varepsilon}\|\mathcal{A}\|^{2} .
$$

Finally, choosing $X=\delta N$, from the estimations in this section we obtain

$$
\begin{aligned}
Q(\mathcal{A})= & (\pi \tan \delta)^{-1} \sum_{m, n} a_{m} a_{n} \sigma(m, n) \\
& +O\left(\left(N+T^{2}+T^{3 / 2} N^{1 / 2}\right) N^{\varepsilon}\|\mathcal{A}\|^{2}\right) .
\end{aligned}
$$

4. Estimation of $R(\mathcal{A})$. From [2] we know that

$$
f_{B}(x)=-\frac{\sinh 2 \beta}{2 \pi} \int_{0}^{1} u x J_{0}(u x)\left(\cosh ^{2} \beta-u^{2}\right)^{-3 / 2} d u \ll \min (x, \sqrt{x}) .
$$

We use this bound only for the terms in $R(\mathcal{A})$ with $|m-n| \leq N T^{-1}$ getting

$$
\begin{aligned}
R_{0}(\mathcal{A}) & =\sum_{|m-n| \leq N T^{-1}} a_{m} a_{n} \sum_{c} c^{-1} S(m, n ; c) f_{B}\left(\frac{4 \pi \sqrt{m n}}{c}\right) \\
& \ll N^{3 / 2+\varepsilon} T^{-1}\|\mathcal{A}\|^{2} .
\end{aligned}
$$

Denote by $R_{1}(\mathcal{A})$ the contribution of terms in $R(\mathcal{A})$ such that $m-n>$ $N T^{-1}$, so $R(\mathcal{A})=R_{0}(\mathcal{A})+2 R_{1}(\mathcal{A})$. We shall transform $R_{1}(\mathcal{A})$ by appealing to $(24)$. First, we evaluate

$$
\widetilde{f}(k-1)=\frac{1}{\pi} \sinh \beta \int_{0}^{\infty} e^{i x \cosh \beta} J_{k-1}(x) d x .
$$

Making use of

$$
\int_{0}^{\infty} e^{-z x} J_{\nu}(x) d x=\left(z^{2}+1\right)^{-1 / 2}\left(\sqrt{z^{2}+1}+z\right)^{-\nu},
$$

where $z=-i \cosh \beta,\left(z^{2}+1\right)^{1 / 2}=-i \sinh \beta$ (see [3]), we get

$$
i^{k} \tilde{f}(k-1)=\frac{1}{\pi} e^{-(k-1) \beta}=\frac{1}{\pi}\left(\frac{n}{m}\right)^{(k-1) / 2} e^{-i \delta(k-1)} .
$$


Thus, by (24) we get

$$
\begin{aligned}
R_{1}(\mathcal{A})= & \frac{1}{\pi^{2}} \sum_{k>0,2 \mid k}(k-1) ! e^{-i \delta(k-1)} \\
& \times \sum_{j} \sum_{m-n>N T^{-1}} a_{m} a_{n}\left(\frac{n}{m}\right)^{(k-1) / 2} \frac{\bar{a}_{j k}(m) a_{j k}(n)}{(4 \pi \sqrt{m n})^{k-1}} .
\end{aligned}
$$

To estimate this we shall use the large sieve inequality (see [1]):

$$
\begin{aligned}
\sum_{2 \leq k \leq K, 2 \mid k} \frac{(k-1) !}{(4 \pi)^{k-1}} \sum_{1 \leq j \leq \theta_{k}}\left|\sum_{N<n \leq 2 N} a_{n} n^{-(k-1) / 2} a_{j k}(n)\right|^{2} \\
\ll\left(K^{2}+N^{1+\varepsilon}\right) \sum_{N<n \leq 2 N}\left|a_{n}\right|^{2} .
\end{aligned}
$$

First observe that the contribution from terms in $R_{1}(\mathcal{A})$ with $k>T N^{\varepsilon}$ is very small since

$$
\left(\frac{n}{m}\right)^{(k-1) / 2}<e^{-(k-1) /(4 T)} .
$$

For the terms with $k \leq T N^{\varepsilon}$ we write

$$
\left(\frac{n}{m}\right)^{(k-1) / 2}=\frac{2}{\pi}(k-1)^{-1} \int_{-\infty}^{\infty}\left(\frac{m}{n}\right)^{i t}\left(1+\left(\frac{2 t}{k-1}\right)^{2}\right)^{-1} d t
$$

by (34) and we remove the condition $m-n>N T^{-1}$ using a Fourier integral (separation of variables). Having done this we apply (45) getting

$$
R_{1}(\mathcal{A}) \ll\left(N+T^{2}\right) N^{\varepsilon}\|\mathcal{A}\|^{2} .
$$

Putting together (44) and (46) we obtain the estimate

$$
R(\mathcal{A}) \ll\left(N+T^{2}+N^{3 / 2} T^{-1}\right) N^{\varepsilon}\|\mathcal{A}\|^{2} .
$$

Finally, inserting (43) and (47) into (30) we get

$$
\begin{aligned}
P(\mathcal{A})= & (\pi \tan \delta)^{-1} \sum_{m, n} a_{m} a_{n} \sigma(m, n) \\
& +O\left(\left(T^{2}+T^{3 / 2} N^{1 / 2}+N^{3 / 2} T^{-1}\right) N^{\varepsilon}\|\mathcal{A}\|^{2}\right) .
\end{aligned}
$$

By (26) this completes the proof of Proposition 2.

5. Evaluation of $T(\mathcal{A})$. We have

$$
\sum_{c} c^{-s} S(0, n ; c)=\frac{1}{\zeta(s)} \sum_{c \mid n} c^{1-s} .
$$


By the same argument as in $[8], \S 3.13$, for $1 / \zeta(s)$, it follows that

$$
\sum_{c} \frac{S(0, n ; c)}{c^{1+i t}}=\sum_{c \leq \exp \left(N^{\varepsilon}\right)} \frac{S(0, n ; c)}{c^{1+i t}}+O\left(N^{-2}\right),
$$

for $t \ll T^{2}$. Since

$$
\eta_{t}(n)=\frac{2 \pi^{1 / 2+i t}}{\Gamma(1 / 2+i t)} n^{i t} \sum_{c} c^{-1-2 i t} S(0, n ; c),
$$

we infer that

$$
\begin{aligned}
\frac{1}{4 \pi} \int_{-\infty}^{\infty} \omega(t) \eta_{t}(n) \bar{\eta}_{t}(m)\left(\frac{m}{n}\right)^{i t} d t \\
=\frac{1}{\pi} \int_{-\infty}^{\infty} \omega(t) \cosh \pi t \\
\quad \times \sum_{c_{1}, c_{2} \leq \exp \left(N^{\varepsilon}\right)}\left(c_{1} c_{2}\right)^{-1}\left(\frac{c_{2}}{c_{1}}\right)^{2 i t} S\left(0, n ; c_{1}\right) S\left(0, m ; c_{2}\right) d t+O\left(N^{-1}\right) \\
=X(m, n)+O\left(N^{-1}\right),
\end{aligned}
$$

say. But ([3])

$$
\int_{-\infty}^{\infty} \frac{\sinh (\pi-2 \delta) t}{\sinh (\pi t)}\left(\frac{c_{2}}{c_{1}}\right)^{2 i t} d t=\frac{2 \sin (2 \delta)}{\left(\frac{c_{2}}{c_{1}}-\frac{c_{1}}{c_{2}}\right)^{2}+(2 \sin \delta)^{2}}
$$

Thus

$$
\begin{aligned}
X(m, n)= & \frac{2 \sin 2 \delta}{\pi} \sum_{c_{1}, c_{2} \leq \exp \left(N^{\varepsilon}\right)}\left(c_{1} c_{2}\right)^{-1}\left(\left(\frac{c_{2}}{c_{1}}-\frac{c_{1}}{c_{2}}\right)^{2}+(2 \sin \delta)^{2}\right)^{-1} \\
& \times S\left(0, n ; c_{1}\right) S\left(0, m ; c_{2}\right) .
\end{aligned}
$$

The terms $c_{1}=c_{2}$ contribute

$$
X_{0}(m, n)=\frac{\cos \delta}{\pi \sin \delta} \sum_{r \leq \exp \left(N^{\varepsilon}\right)} r^{-2} S(0, m ; r) S(0, n ; r) .
$$

From the elementary inequalities

$$
\begin{aligned}
|S(0, n ; r)| & \leq(n, r) d(n), \\
\sum_{c \leq D}\left|\sum_{n \leq N} a_{n}(n, c)\right|^{2} & \ll(D N)^{1+\varepsilon} \sum_{n \leq N}\left|a_{n}\right|^{2},
\end{aligned}
$$


we obtain

$$
\sum_{m, n} a_{m} a_{n} X_{0}(m, n)=(\pi \tan \delta)^{-1} \sum_{m, n} a_{m} a_{n} \sigma(m, n)+O\left(T^{2}\|\mathcal{A}\|^{2}\right) .
$$

The terms $c_{1}<c_{2}$ contribute

$$
\begin{aligned}
& X_{1}(m, n) \\
& =\frac{2 \sin 2 \delta}{\pi} \sum_{c_{1}<\exp \left(N^{\varepsilon}\right)} c_{1} S\left(0, n ; c_{1}\right) \sum_{c_{1}<c_{2} \leq \exp \left(N^{\varepsilon}\right)} c_{2}^{-1} S\left(0, m ; c_{2}\right) \gamma\left(c_{1}, c_{2}\right),
\end{aligned}
$$

where

$$
\gamma\left(c_{1}, c_{2}\right)=\left[\left(c_{2}-c_{1}\right)^{2}\left(1+\frac{c_{1}}{c_{2}}\right)^{2}+\left(2 c_{1} \sin \delta\right)^{2}\right]^{-1}
$$

Using (50), (51) and considering the following cases separately: $c_{1} \leq T$; $c_{2}-c_{1}>c_{1} / T>1 ; c_{2}-c_{1} \leq c_{1} / T>1$, we deduce that

$$
\sum_{m, n} a_{m} a_{n} X_{1}(m, n) \ll\left(N+T^{2}\right) N^{\varepsilon}\|\mathcal{A}\|^{2} .
$$

By (52) and (53) we complete the proof of Proposition 1. Both Propositions 1 and 2 give Theorem 1 as shown in the first section.

\section{References}

[1] J.-M. Deshouillers and H. Iwaniec, Kloosterman sums and Fourier coefficients of cusp forms, Invent. Math. 70 (1982), 219-288.

[2] - - - The non-vanishing of Rankin-Selberg zeta-functions at special points, in: The Selberg Trace Formula and Related Topics, Contemp. Math. 53, Amer. Math. Soc., Providence, R.I., 1986, 59-95.

[3] I. S. Gradshtein and I. M. Ryzhik, Tables of Integrals, Series and Products, Academic Press, New York and London, 1965.

[4] D. A. Hejhal, The Selberg Trace Formula for PSL(2, R), Vol. 2, Lecture Notes in Math. 1001, Springer, 1983.

[5] M. N. Huxley, The large sieve inequality for algebraic number fields. II: Means of moments of Hecke zeta-functions, Proc. London Math. Soc. (3) 21 (1970), 108-128.

[6] H. Iwaniec, Fourier coefficients of cusp forms and the Riemann zeta-function, Séminaire de Théorie des Nombres, Bordeaux 1979-80.

[7] M. Jutila, On spectral large sieve inequalities, preprint, 1991.

[8] N. V. Kuznetsov, Petersson's conjecture for cusp forms of weight zero and Linnik's conjecture. Sums of Kloosterman sums, Mat. Sb. 111 (1980), 334-383 (in Russian).

[9] W. Luo, On the non-vanishing of Rankin-Selberg L-functions, Duke Math. J. 69 (1993), 411-425.

[10] H. L. Montgomery, Topics in Multiplicative Number Theory, Lecture Notes in Math. 227, Springer, 1971.

[11] R. Phillips and P. Sarnak, On cusp forms for cofinite subgroups of PSL(2,R), Invent. Math. 80 (1985), 339-364. 
[12] R. A. Rankin, Contributions to the theory of Ramanujan's function $\tau(n)$ and similar arithmetic functions, Proc. Cambridge Philos. Soc. 35 (1939), 357-372.

[13] A. Selberg, On the estimation of Fourier coefficients of modular forms, in: Proc. Sympos. Pure Math. 8, Amer. Math. Soc., Providence, R.I., 1965, 1-15.

[14] - Collected Papers, Vol. 1, Springer, 1989, 626-674.

[15] G. Shimura, Introduction to the Arithmetic Theory of Automorphic Functions, Princeton Univ. Press, 1971.

[16] E. C. Titchmarsh, The Theory of the Riemann Zeta-Function, Oxford, 1951.

[17] G. N. Watson, A Treatise on the Theory of Bessel Functions, Cambridge, 1944.

MATHEMATICAL SCIENCES RESEARCH INSTITUTE

1000 CENTENNIAL DRIVE

BERKELEY, CALIFORNIA 94720

U.S.A.

Received on 17.2.1993

and in revised form on 25.4.1994 